\title{
Effect of Shock Proximity on Richtmyer-Meshkov Growth
}

S.G. Glendinning, J. Bolstad, D.G. Braun, M.J. Edwards, W.W. Hsing, B.F. Lasinski, H. Louis, A. Miles, J. Moreno, T.A. Peyser, B.A. Remington, H.F. Robey, E.J. Turano, C.P. Verdon, and Y. Zhou

This article was submitted to the $44^{\text {th }}$ Annual Meeting of the Division of Plasma Physics, Orlando, Florida, November 11-15, 2002

Lawrence

October 31, 2002

Livermore

National

Laboratory 


\section{DISCLAIMER}

This document was prepared as an account of work sponsored by an agency of the United States Government. Neither the United States Government nor the University of California nor any of their employees, makes any warranty, express or implied, or assumes any legal liability or responsibility for the accuracy, completeness, or usefulness of any information, apparatus, product, or process disclosed, or represents that its use would not infringe privately owned rights. Reference herein to any specific commercial product, process, or service by trade name, trademark, manufacturer, or otherwise, does not necessarily constitute or imply its endorsement, recommendation, or favoring by the United States Government or the University of California. The views and opinions of authors expressed herein do not necessarily state or reflect those of the United States Government or the University of California, and shall not be used for advertising or product endorsement purposes.

This is a preprint of a paper intended for publication in a journal or proceedings. Since changes may be made before publication, this preprint is made available with the understanding that it will not be cited or reproduced without the permission of the author.

This report has been reproduced directly from the best available copy.

Available electronically at http://www.doc.gov/bridge

Available for a processing fee to U.S. Department of Energy

And its contractors in paper from

U.S. Department of Energy

Office of Scientific and Technical Information

P.O. Box 62

Oak Ridge, TN 37831-0062

Telephone: (865) 576-8401

Facsimile: (865) 576-5728

E-mail: reports@adonis.osti.gov

Available for the sale to the public from

U.S. Department of Commerce

National Technical Information Service

5285 Port Royal Road

Springfield, VA 22161

Telephone: (800) 553-6847

Facsimile: (703) 605-6900

E-mail: orders@ntis.fedworld.gov

Online ordering: http://www.ntis.gov/ordering.htm

\section{OR}

Lawrence Livermore National Laboratory

Technical Information Department's Digital Library

http://www.llnl.gov/tid/Library.html 


\title{
Effect of Shock Proximity on Richtmyer-Meshkov Growth
}

S.G.Glendinning, J.Bolstad, D.G.Braun, M.J.Edwards, W.W.Hsing, B.F.Lasinski, H.Louis, A. Miles, J.Moreno, T.A.Peyser, B.A.Remington, H.F.Robey, E.J.Turano, C.P.Verdon, Y.Zhou Lawrence Livermore National Laboratory, Livermore, California 94550

\begin{abstract}
We report here experiments, conducted on the Omega laser [T.R.Boehly et al., Optics Commun. 133, 495 (1997)], and simulations that show reduced RichtmyerMeshkov growth rates in a strongly shocked system with linear initial amplitudes $\left(\mathrm{k}_{0} \leq 0.9\right)$. The growth rate at early time is less than half the impulsive model prediction, rising at later time to near the impulsive prediction. An analytical model that accounts for shock proximity agrees with the results.
\end{abstract}

A strong shock (shock pressure much greater than pressure ahead of the shock) in a material results in the maximum single-shock compression of the material. For a gamma-law ideal gas, the maximum compression is $(\gamma+1) /(\gamma-1)$, or a compression of four for a typical $\gamma=5 / 3$ gas. When this strong shock reaches an interface between two densities, the high compression will result in a transmitted shock that recedes slowly from the interface. Impulsive theory of the RichtmyerMeshkov (RM) instability, ${ }^{1,2}$ predicts a nonzero initial growth rate, which may be larger than the shock recession velocity. Linear compressible theory of Yang, Zhang, and Sharp (YZS) ${ }^{3}$, which should provide a better description for strong shocks, predicts that a perturbation at the interface will have an initial growth rate of zero, followed by a period of interaction between the rippled transmitted 
shock and the growing interface modulation. Several researchers ${ }^{48}$ have proposed that the shock will represent an impenetrable boundary, reducing the growth rate. The reduction would be enhanced in a case where the shock goes from high to low density, as the high-density spikes of material have a higher growth rate than the low-density bubbles ${ }^{9}$. The predicted incompressible linear growth rate in this instance is usually taken to be that of Meyer and Blewett (MB) 10,

$$
u_{I M}=A^{+} k u_{c}\left(\frac{\eta_{0}+\eta^{+}}{2}\right)
$$

where $\mathrm{u}_{\mathrm{IM}}$ is the incompressible linear growth rate, $\mathrm{A}^{+}$is the post-shock Atwood number, $k$ is the wave number, $\eta_{0}$ is the initial amplitude, $\eta^{+}$is the post-shock amplitude, and $u_{c}$ is the interface velocity. It is not difficult to find configurations in which this growth rate would exceed the transmitted shock velocity.

Researchers have suggested using possible shock proximity corrections to the impulsive growth rate, including Holmes':

$$
u=\frac{u_{I M}}{1+u_{I M} /\left(s_{t}-u_{c}\right)}
$$

and Hurricane ${ }^{7}$

$$
u=u_{c}\left(1-u_{c} / s_{t}\right) \tanh \left[\frac{u_{I M}}{u_{c}\left(1-u_{c} / s_{t}\right)}\right]
$$


where $u$ is the growth rate and $s_{t}$ is the transmitted shock velocity. These growth rates are fits to numerical simulations of growth in systems expected to be compressible. Neither of these models predicts a change in the growth rate as the shock recedes and allows the perturbation to grow at higher rates. However, linear compressible theory ${ }^{3}$ shows that the initial growth rate in all cases is theoretically zero, with the growth rate increasing as the shock recedes. The growth for the different incompressible and compressible models is shown in Figure 1. The ratio of the incompressible growth rate to the shock recession velocity, $\varepsilon=u_{I M} /\left(s_{t}-u_{c}\right)$, may serve as an estimate of the effect of compressibility; where $\varepsilon$ is close to zero, the system may be described by impulsive models, but as $\varepsilon$ approaches or exceeds unity, one might expect compressible theory to be necessary. The results using the YZS theory are shown in Figure 1 for two values of $\varepsilon, 0.13$ and 1.2. The lower value of $\varepsilon$ is well described by the linear impulsive model, while the higher value is not. Also shown in Figure 1 is the nonlinear, impulsive prediction of Sadot ${ }^{11}$. Sadot et al. have proposed an incompressible model, describing existing shock tube data very well ${ }^{12}$, in which the growth rate decreases as a function of time, as

$$
u(t)=u_{L M} \frac{1+u_{\mathrm{IM}} k t}{1+\left(1 \pm A^{*}\right) u_{\mathrm{LM}} k t+\frac{1}{2 \pi C} \frac{1 \pm A^{*}}{1+A^{*}}\left(u_{\mathrm{IM}} k t\right)^{2}}
$$

where the plus signs are for bubble velocity, the minus signs are for spike velocity, and $C$ is $1 / 3 \pi$ for $A^{*}>0.5$ and $1 / 2 \pi$ for $A^{*} \rightarrow 0$. It is clear from this model that the range of validity of the linear models is small. By the time the normalized amplitude $\mathrm{kh}$ has reached 0.5 , the error in using a linear model is 
already $20 \%$. A model which includes both nonlinear and compressible effects would extend the predictive capabilities of RM models. Analytical models ${ }^{13,14}$ that describe the RM instability as a line of vortices have been shown to correctly predict shock tube data for low Atwood numbers well into the nonlinear regime. The models assume an Atwood number of zero. Such models have been extended by Robey et al. ${ }^{15}$ to include the effect of shock proximity by treating the shock as a rigid boundary condition. The line of vortices is reflected across the shock position, giving zero growth at the shock position, and reducing the growth in the solution found for the density interface. As the shock recedes, the effect of the image vortices is diminished, until the growth rate recovers to higher rates, as predicted by YZS, but including the effect of nonlinearity. The growth is described by the expression

$$
u=\frac{-k \Gamma}{\pi}\left\{\frac{\cosh (k y)}{1+\cosh (2 k y)}-\frac{\cosh \left[k\left(2\left(\eta^{+}+\Delta s_{t} t\right)-y\right)\right]}{1+\cosh \left[k\left(2\left(\eta^{+}+\Delta s_{t} t\right)-y\right)\right]}\right\}
$$

where $\Gamma$ is the magnitude of the vortex strength $\left(=2 \pi \mathrm{u}_{\mathrm{IM}} / \mathrm{k}\right), \mathrm{y}$ is the displacement of the interface in the direction of shock propagation, and $\Delta \mathrm{s}_{\mathrm{t}}$ is the shock recession velocity $\left(\mathrm{s}_{\mathrm{t}}-\mathrm{u}_{\mathrm{c}}\right)$.

Previously, experiments have been performed using lasers to examine the effect of compressibility at high Mach number. The data of Dimonte et al., ${ }^{4,5}$ and Farley et al. ${ }^{8}$ showed reduced growth rates (compared with incompressible 
predictions), qualitatively in agreement with the models of Holmes and Hurricane. However, these experiments used large initial amplitudes $\left(k \eta_{0} \sim 2\right)$ to achieve predicted high initial growth rates. It has been proposed by Velikovich et al. ${ }^{16}$ and demonstrated by Sadot et al., ${ }^{97}$ that high initial amplitudes reduce the growth rate, even for incompressible systems, as shown in Figure 2. This makes the interpretation of the data of Dimonte and Farley difficult, as much of the observed reduction in growth rate would be attributed to a large initial amplitude. Velikovich predicts a reduction factor of 0.6 for $k \eta_{0} \sim 2$. Rikanati ${ }^{9}$ predicts a reduction by 0.65 and a Mach 15 system such as those of Dimonte and Farley. For the large amplitude effect to be less than about $10 \%$, the initial conditions would require $\mathrm{k}_{0} \leq 0.9$.

If the interface velocity is not steady but is decreasing, a significant amount of growth may be due to the Rayleigh-Taylor (RT) instability ${ }^{18,19}$, which is exponential rather than linear in time. We simulated the effect on amplitude growth of an interface velocity which would decrease by about $30 \%$ during the measurement. The amplitude growth increased by about $20 \%$, as shown in Figure 3. RT growth in the experiment larger than $10 \%$ will certainly confuse interpretation of the results. Thus, experiments to test only compressibility effects should have: 1) a predicted incompressible growth rate comparable to the shock recession velocity $(\varepsilon \sim 1), 2)$ an initial amplitude $<0.9 / k$, and 3) RT growth of less than $10 \%$. The experiments reported in this paper were designed to meet these requirements. 
The experimental configuration for our experiment is shown in Figure 4. The target was a cylindrical package $800 \mu \mathrm{m}$ in diameter composed of several layers. The first layer, which was ablated by the incident laser beams, was $20 \mu \mathrm{m}$ of polycarbonate (density $1.2 \mathrm{~g} / \mathrm{cm}^{3}$ ). The second layer was $170 \mu \mathrm{m}$ of brominated polystyrene $\left(\mathrm{C}_{50} \mathrm{H}_{48} \mathrm{Br}_{2}\right.$, density $\left.1.23 \mathrm{~g} / \mathrm{cm}^{3}\right)$, designed to shield the interface from $\mathrm{x}$-rays created at the ablation front. The third layer was a composite, $50 \mu \mathrm{m}$ thick, with a $200 \mu \mathrm{m}$ wide stripe of brominated polystyrene running perpendicular to the line of sight, surrounded by polycarbonate. The purpose of this stripe was to concentrate the opacity near the center of the target to minimize edge effects. This layer was modulated on the side away from the laser drive with a sinusoidal perturbation of wavelength $150 \mu \mathrm{m}$, initial amplitude either 7 or 22 $\mu \mathrm{m}$. The modulated plastic layer was in contact at its peaks with a carbonized resorcinol formaldehyde foam (CRF) payload of density $0.1 \mathrm{~g} / \mathrm{cm}^{3}$. The CRF foam layer was not modulated, due to the additional fabrication cost and difficulties.

The primary diagnostic technique used in this experiment was radiography. $\mathrm{X}$ rays for radiography were produced by ten beams of the Omega laser incident on a thin foil of Scandium, at about $8 \times 10^{14} \mathrm{~W} / \mathrm{cm}^{2}$, in a spot $600 \mu \mathrm{m}$ in diameter. The beams each delivered $500 \mathrm{~J}$ in a $1 \mathrm{~ns}$ square pulse and were timed in two groups $4 \mathrm{~ns}$ apart. The emission is predominantly He- $\alpha$ at $4.3 \mathrm{keV}^{20}$. The foil was oriented perpendicular to the motion of the interface, and was imaged by a gated $x$-ray pinhole camera ${ }^{21}$. The imager was an array of 16 pinholes, each $10 \mu \mathrm{m}$ diameter, providing 16 images at four different times (two groups $4 \mathrm{~ns}$ apart). A typical frame $20 \mathrm{~ns}$ after the start of the laser drive is shown in Figure 4. On some 
shots the target was unmodulated and the imaging was done with an x-ray streak camera, giving the position of the interface as a function of time.

The laser drive was provided by nine beams of the Omega laser, each smoothed with a distributed phase plate ${ }^{22}$ into a superGaussian shape of the form exp[$\left.(r / d)^{n}\right]$, where $r$ is the distance from the beam axis, $d$ is $430 \mu \mathrm{m}$, and $n$ is 4.7. The angles of incidence were $47.8^{\circ}$ for six of the beams and $23.2^{\circ}$ for three of the beams. The beams were used in sets of 3 (two $47.8^{\circ}$ sets followed by one $23.2^{\circ}$ set), with each set $3.7 \mathrm{~ns}$ later than the set before. The pulse length was nominally $3.7 \mathrm{~ns}$, providing a nearly constant $11.1 \mathrm{~ns}$ drive. The energy was nominally 250 $\mathrm{J} /$ beam in the $47.8^{\circ}$ sets and $190 \mathrm{~J} /$ beam in the $23.2^{\circ}$ set, providing an average intensity of $2.6 \times 10^{13} \mathrm{~W} / \mathrm{cm}^{2}$, as shown in Figure 5 (dashed curve, right hand axis). A target with the payload removed was imaged with a VISAR ${ }^{23,24}$ diagnostic on separate shots to determine the incident shock velocity. The velocity inferred from the VISAR is also shown in Figure 5 (solid curve, left hand axis).

The two-dimensional radiation hydrodynamics code LASNEX ${ }^{25}$ was used to model the laser deposition in the target, the propagation of the shock through the pusher, and the subsequent (unperturbed) interface motion. The LASNEX simulation of the shock velocity as a function of time is shown in Figure 5 . The simulations and the data show a shock velocity that is constant to $\pm 5 \%$ RMS, with some temporal structure due to structure in the laser pulse. LASNEX predicted an average interface velocity of $21.0 \mu \mathrm{m} / \mathrm{ns}$, with $\pm 5 \%$ RMS fluctuations, an average transmitted shock speed of 26.4, an average post-shock Atwood number 
of about 0.5 , and a Mach number for the incident shock of about 10. From streak camera images of the unmodulated interface and transmitted shock, we extracted an average velocity of $22 \pm 1 \mu \mathrm{m} / \mathrm{ns}$ for the interface and $26 \pm 1 \mu \mathrm{m} / \mathrm{ns}$ for the transmitted shock.

Images obtained by the side-on radiography were analyzed to determine the spike-to-bubble distance. As the experiment does not allow us to separate the spike and bubble amplitudes with sufficient precision to tell any difference, we have used $1 / 2$ the spike-to-bubble distance as the average amplitude. The results are plotted in Figure 6 for both the $7 \mu \mathrm{m}$ initial amplitude and the $22 \mu \mathrm{m}$ initial amplitude. Also shown in Figure 6 is the linear, compressible theory prediction of YZS for both data sets. YZS is close to the $7 \mu \mathrm{m}$ initial amplitude data, but predicts significantly more growth than is observed in the $22 \mu \mathrm{m}$ initial amplitude data (about 55\% more growth at $24 \mathrm{~ns}$ ).

Using the two-dimensional radiation hydrodynamics code $\mathrm{CALE}^{26}$, we simulated the growth for both the actual laser drive and a constant velocity drive. The initial gap between the valleys of the modulated plastic and the unmodulated foam was included in the simulations. The actual laser drive was simulated in 2dimensional LASNEX in the same way it was for the VISAR shots. The velocity of a zone $20 \mu \mathrm{m}$ into the pusher was extracted from the LASNEX simulations, and used as a velocity source in the CALE simulations. A constant velocity source at the time-averaged velocity $(14.3 \mu \mathrm{m} / \mathrm{ns})$ for the zone was also used. The constant velocity drive and the actual laser drive results are shown in Figure 6; both are shown offset vertically by about $-4 \mu \mathrm{m}$ to agree with the post-shock 
amplitude from the data. Both simulations predict a larger effect of the gap than is observed in the data. We believe that this difference and the other small remaining differences between the simulation and the data result from the exact timing of the small shocks arriving at the interface. The difference between the constant drive and actual drive is less than about $3 \mu \mathrm{m}$ in amplitude. Little RT growth was found in our simulations. The simulation of the interface velocity showed a decrease in velocity of $12 \%$ by the end of the experiment. From Figure 3 , we might expect an RT component of the growth of about $5 \%$ by the end of the measurement.

In order to compare RM growth for different wavelengths, initial amplitudes, and linear growth rates, a dimensionless normalization of the data is convenient. The data are offset by the post-shock amplitude and normalized to the wave number, and the experimental times are offset by the post-shock time and normalized to the impulsive (MB) predicted amplitude. The post-shock amplitude is usually the Richtmyer amplitude, $\eta^{+}=\left(1 .-u_{c} / s_{i}\right) \eta_{0}$, and the postshock time is the time at which the shock leaves the initial perturbation. However, our experiments used targets in which the modulation is only in the pusher; there is no foam initially in the spike locations. There is thus a brief interval ( $2 \mathrm{~ns}$ in the $22 \mu \mathrm{m}$ data) of increased growth in the spike locations, resulting in a post-shock amplitude greater than the Richtmyer amplitude. We have therefore offset our $22 \mu \mathrm{m} \eta_{0}$ modeling to the amplitude at the time the shock leaves the initial perturbation ( $5 \mu \mathrm{m}$ in agreement with the data). For the 7 $\mu \mathrm{m}$ data we used an offset of $1.6 \mu \mathrm{m}$. The initial time offset is taken from the 
CALE simulations, $12.4 \mathrm{~ns}$ for the $22-\mu \mathrm{m}$ data and $11.7 \mathrm{~ns}$ for the $7-\mu \mathrm{m}$ data. The amplitudes are then scaled by the wave number and the time is normalized to the product of the wavenumber and the impulsive growth rate, $\mathrm{u}_{\mathrm{IM}}(5.6 \mu \mathrm{m} / \mathrm{ns}$ for the $22 \mu \mathrm{m}$ data and $1.8 \mu \mathrm{m} / \mathrm{ns}$ for the $7 \mu \mathrm{m}$ data). The resulting scaled data are shown in Figure 7. Predictions from four analytical models are also shown. First is the incompressible, linear growth as predicted by the Meyer-Blewett impulsive model (solid curve). As all plots are normalized to the MB growth rate, this curve is a straight line with a slope of one. As the amplitude increases, the growth leaves the linear regime, as shown by the Sadot model (dash-dot curve). The impulsive predictions are the same any initial amplitudes or wavelength. We note that $\mathrm{MB}$ would predict about $60 \%$ more growth than the Sadot model at the largest $\mathrm{k \eta}$, similar to the difference between YZS theory and the data for the 22 $\mu \mathrm{m}$ initial amplitude result.

The 7- $\mu \mathrm{m}$ data are within one sigma of the Sadot incompressible prediction, although all points are low early in the sequence. One might expect an incompressible model to do a fairly good job on the $7 \mu \mathrm{m}$ data, as $\mathrm{u}_{\mathrm{IM}}(1.8 \mu \mathrm{m} / \mathrm{ns})$ is significantly less than the shock recession velocity $(\varepsilon=0.4)$. As expected, the 22$\mu \mathrm{m}$ data does not agree with either incompressible model. The normalized predictions of the image vortex model are also shown (dashed and dotted curves) for the two different initial amplitudes. The image vortex model predicts different normalized results for the two initial amplitudes, as is observed in the data. The $22-\mu \mathrm{m}$ data agree with the image vortex model, while the $7-\mu \mathrm{m}$ data are slightly higher. It might seem surprising that a model requiring an Atwood 
number of zero does a reasonably good job of describing an experiment with an Atwood number of about 0.5 . However, the shock proximity retards the growth of spikes more than it does the growth of bubbles. Thus the spikes and bubbles are nearly symmetric (as predicted by Holmes), much as for a very low Atwood number, as may be seen in the image in Figure $4 \mathrm{c}$, and the growth has the symmetry required for the vortex model.

In conclusion, we have performed a high Mach number shock instability experiment, not complicated by a very large initial amplitude nor by significant Rayleigh-Taylor growth. Because incompressible models (Meyer-Blewett and Sadot) predict an initial perturbation growth rate which exceeds the shock recession velocity, they do not correctly predict the observed growth. Models such of those of Holmes et al. and Hurricane et al. which reduce the initial growth rate and assume a monotonically decreasing growth rate thereafter correctly predict the average growth, but do not correctly predict the growth rate as a function of time. Linear compressible theory of Yang, Zhang, and Sharp, does not account for the nonlinear evolution of the perturbation growth. A model based on vortex evolution, although assuming a zero Atwood number, does a good job of predicting observed growth. 


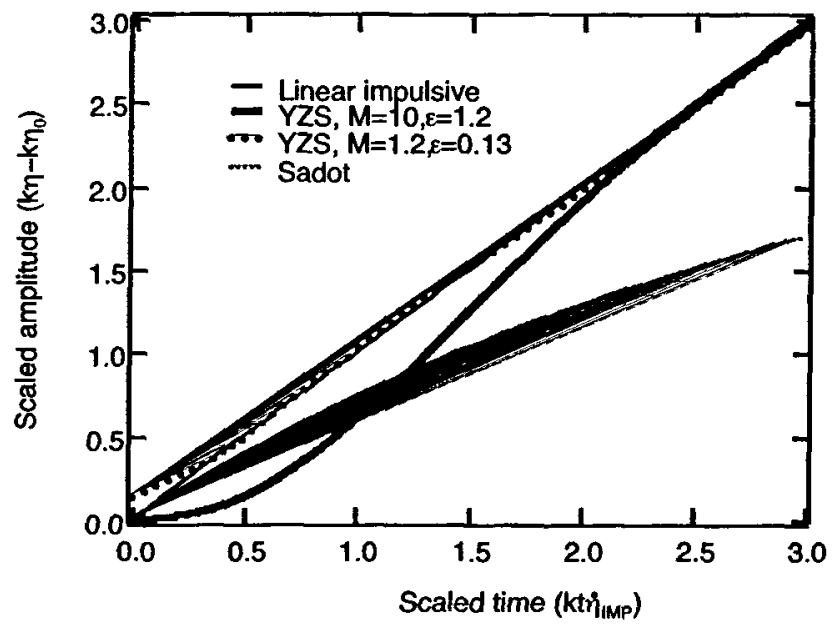

Figure 1. Predictions of various models of the RM instability. The axes are normalized amplitude (wave number times amplitude minus the post-shock amplitude) vs. normalized time (wave number times amplitude from the MeyerBlewett impulsive model). The MB impulsive model is the light solid line, the Sadot impulsive nonlinear model is the light dotted line, and two predictions of the YZS theory (for $\varepsilon=0.13$ and 1.2) are shown as the heavy dashed and heavy solid lines. 


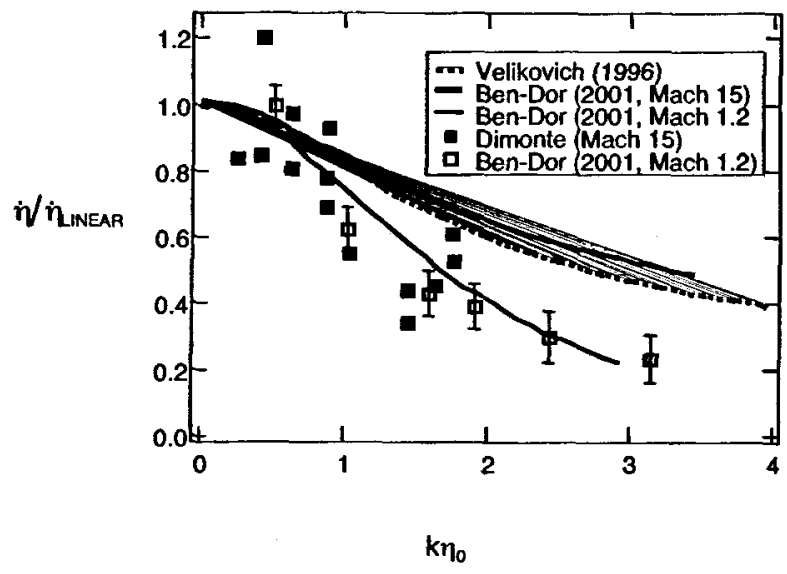

Figure 2. Effect of large initial amplitude on growth rate. Impulsive models of Velikovich for Mach number 15 is shown as the dashed line and Rikanati for Mach numbers 1.2 and 15 are the heavy and light solid lines, respectively. The data of Dimonte is shown as solid squares and that of Sadot as open squares. 

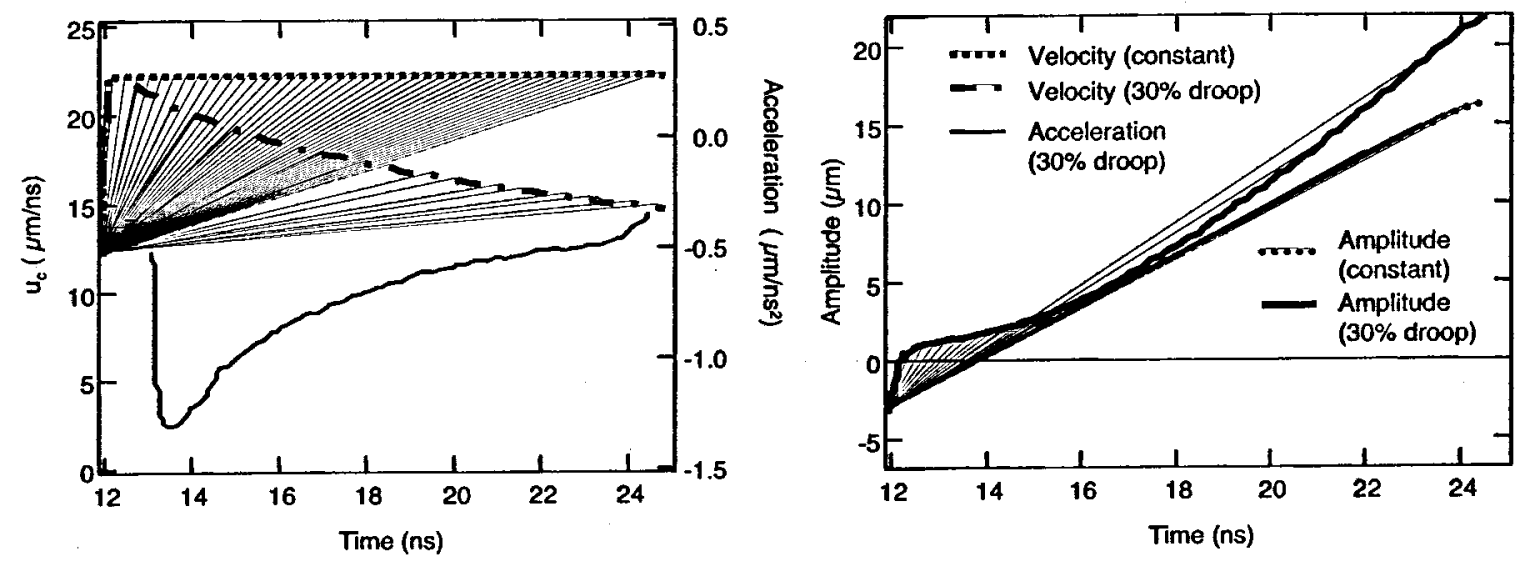

Figure 3. Effect of deceleration on perturbation growth. A) Constant velocity (dashed) and decelerating velocity (dot-dash) as a function of time, both on left hand axis, and acceleration as a function of time for the decelerating velocity (thin solid curve, right hand axis). B) Calculated amplitude as a function of time for constant (dotted) and decelerating (heavy solid curve) velocities. 
a)

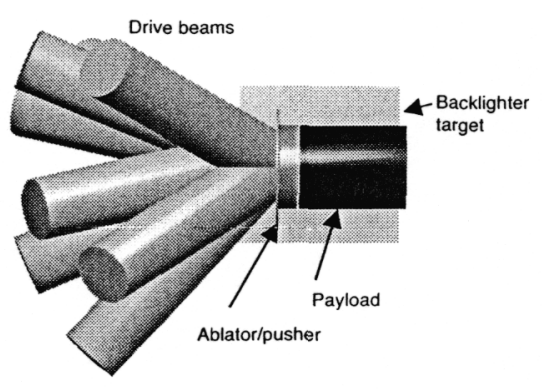

b)

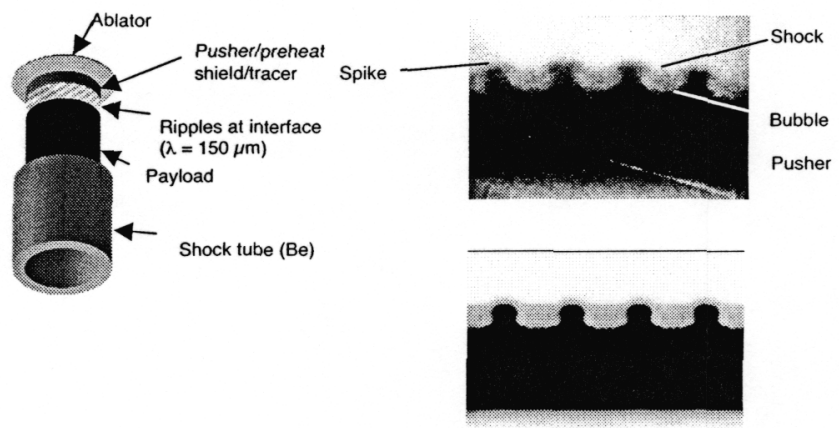

Figure 4. a) Experimental schematic. Drive laser beams are incident from the left on the polycarbonate ablator. b) Exploded view of package, showing polycarbonate ablator, brominated polystyrene pusher/preheat shield, highopacity tracer stripe embedded in polycarbonate with machined ripples, CRF payload, and beryllium shock tube. c) Typical radiograph image (top) showing the shock front (moving up), spikes of pusher material (dark) moving into payload material (light), bubbles of payload material moving into pusher material, and (bottom) a simulation of the radiograph, both $20 \mathrm{~ns}$ after the start of the laser drive. The spikes are separated by $150 \mu \mathrm{m}$. 


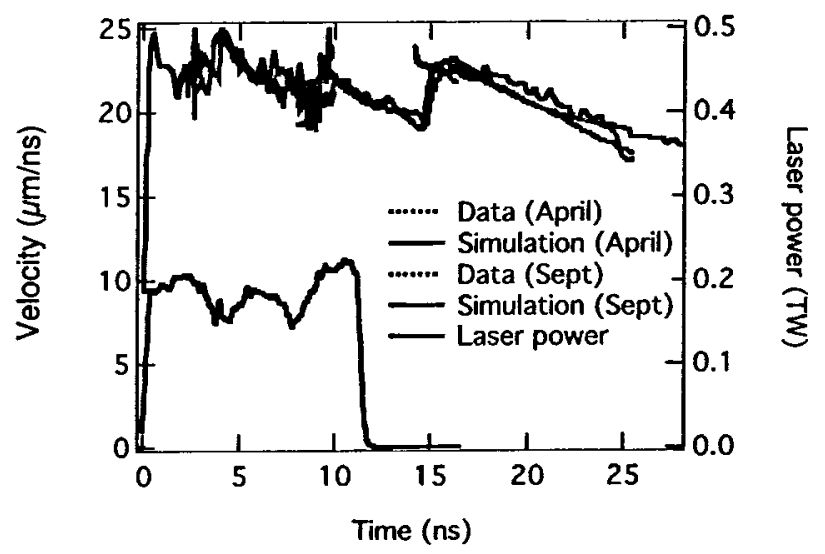

Figure 5. Incident shock velocity (measured and simulated) and drive laser pulse. 


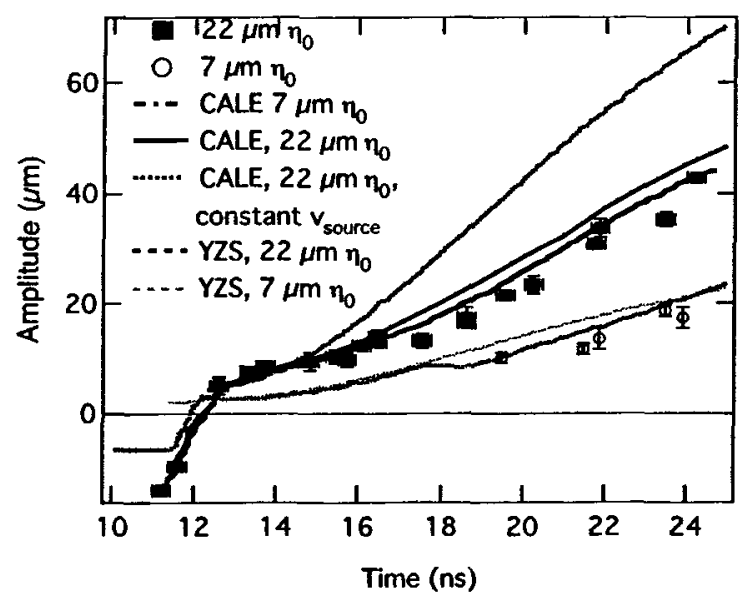

Figure 6. Modulation growth as a function of time for two initial amplitudes, 22 $\mu \mathrm{m}$ (squares) and $7 \mu \mathrm{m}$ (circles). CALE simulations for the data using a constant velocity source (dashed) and the velocity source calculated by 2D LASNEX (solid) are also shown. The linear theory of Yang, Zhang, and Sharp is shown for the $7 \mu \mathrm{m}$ data (thin dashed line) and the $22 \mu \mathrm{m}$ data (thick dashed line). 


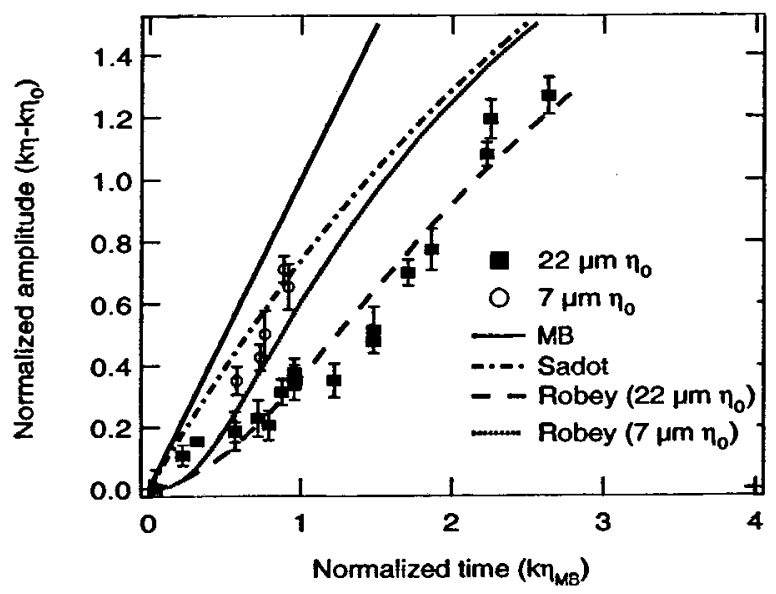

Figure 7. Normalized modulation growth as a function of normalized time for two initial amplitudes, $22 \mu \mathrm{m}$ (squares) and $7 \mu \mathrm{m}$ (circles). The growth predicted by the linear impulsive (Meyer-Blewett), saturated impulsive (Sadot), and image vortex (Robey) models are also shown. 
1 R. D. Richtmyer, Communications on Pure and Applied Mathematics 13, $297(1960)$

2 Y. Y. Meshkov, Izvestiya Akademii Nauk SSSR, Mekhanika Zhidkosti i Gaza, 151 (1969).

3

Y. M. Yang, Q. A. Zhang, and D. H. Sharp, Physics of Fluids 6, 1856 (1994).

G. Dimonte and B. Remington, Physical Review Letters 70, 1806 (1993).

G. Dimonte, C. E. Frerking, M. Schneider, et al., Physics of Plasmas 3, 614 (1996).

R. L. Holmes, G. Dimonte, B. Fryxell, et al., Journal of Fluid Mechanics 389, 55 (1999).

O. A. Hurricane, E. Burke, S. Maples, et al., Physics of Fluids 12, 2148 (2000).

D. R. Farley, T. A. Peyser, L. M. Logory, et al., Physics of Plasmas 6, 4304 (1999).

G. BenDor, I. Ozer, and T. Elperin, Handbook of Shock Waves (Academic Press, London, 2001).

K. A. Meyer and P. J. Blewett, Physics of Fluids 15, 753 (1972).

O. Sadot, L. Erez, U. Alon, et al., Physical Review Letters 80, 1654 (1998).

J. W. Jacobs, (University of Arizona, 2000), p. 18.

J. W. Jacobs and J. M. Sheeley, Physics of Fluids 8, 405 (1996).

4 A. Rikanati, U. Alon, and D. Shvarts, Physical Review E 58, 7410 (1998).

H. F. Robey, J. Bolstad, S. G. Glendinning, et al., Physics of Fluids (submitted) (2002). 
A. L. Velikovich and G. Dimonte, Physical Review Letters 76, 3112 (1996).

O. Sadot, A. Yosef-Hai, A. Rikanati, et al., Bulletin of the American

Physical Society 44, 57 (1999).

18 J. W. S. Rayleigh, Scientific Papers (University press, Cambridge, 1899).

19 G. I. Taylor, Proceedings of the Royal Society A201, 192 (1950).

20 S. G. Glendinning, O. L. Landen, B. A. Remington, et al., in Applications of Laser Plasma Radiation II (SPIE, Bellingham, WA, 1995), Vol. 2523, p. 29.

${ }^{21} \quad$ K. S. Budil, T. S. Perry, P. M. Bell, et al., Review of Scientific Instruments 67, 485 (1996).

22 Y. Lin, T. J. Kessler, and G. N. Lawrence, Optics Letters 20, 764 (1995).

23 L. M. Barker and R. E. Hollenbach, Journal of Applied Physics 43, 4669 (1972).

24 L. M. Barker and K. W. Schuler, Journal of Applied Physics 45, 3692 (1974).

G. B. Zimmerman and W. L. Kruer, Comments on Plasma Physics and Controlled Fusion 2, 51 (1975). 
Figure 1.

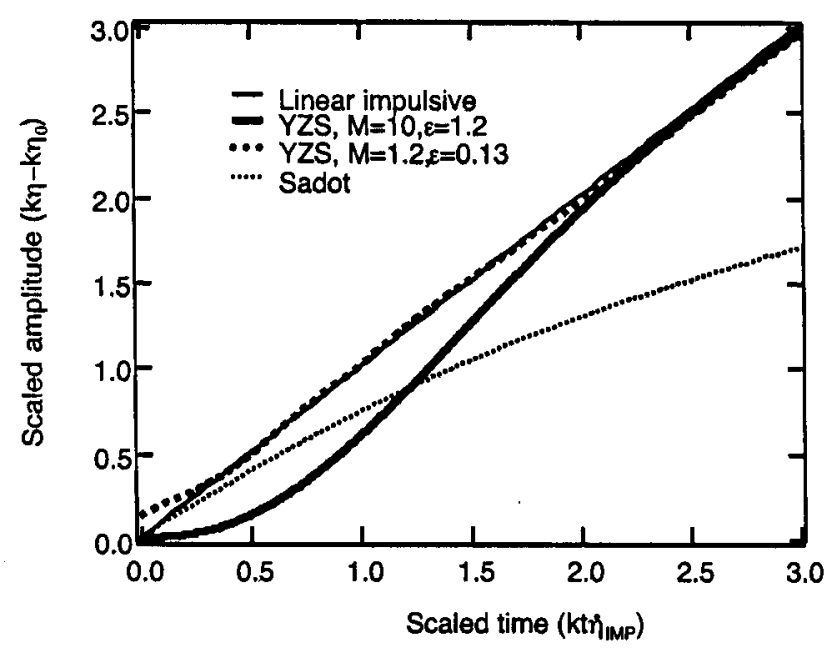

sgg-281002-01 
Figure 2.

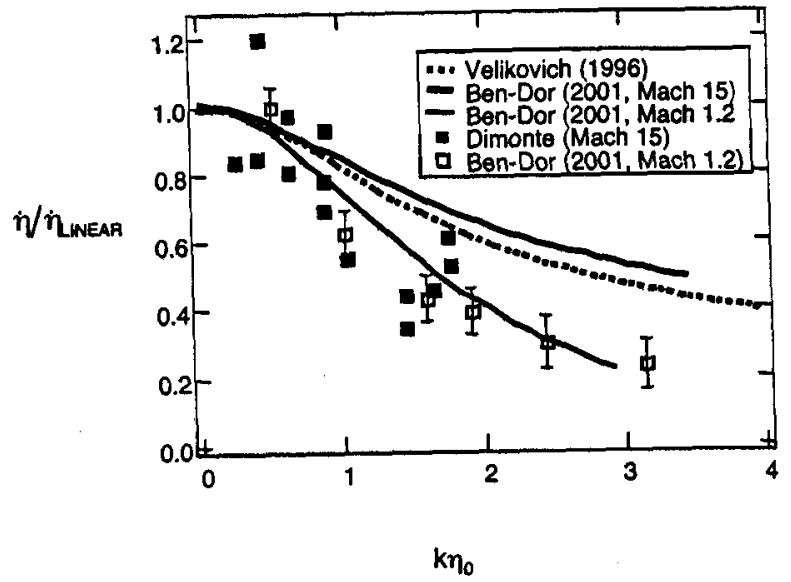

- $\quad$ sgg-281002-01 
Figure 3.
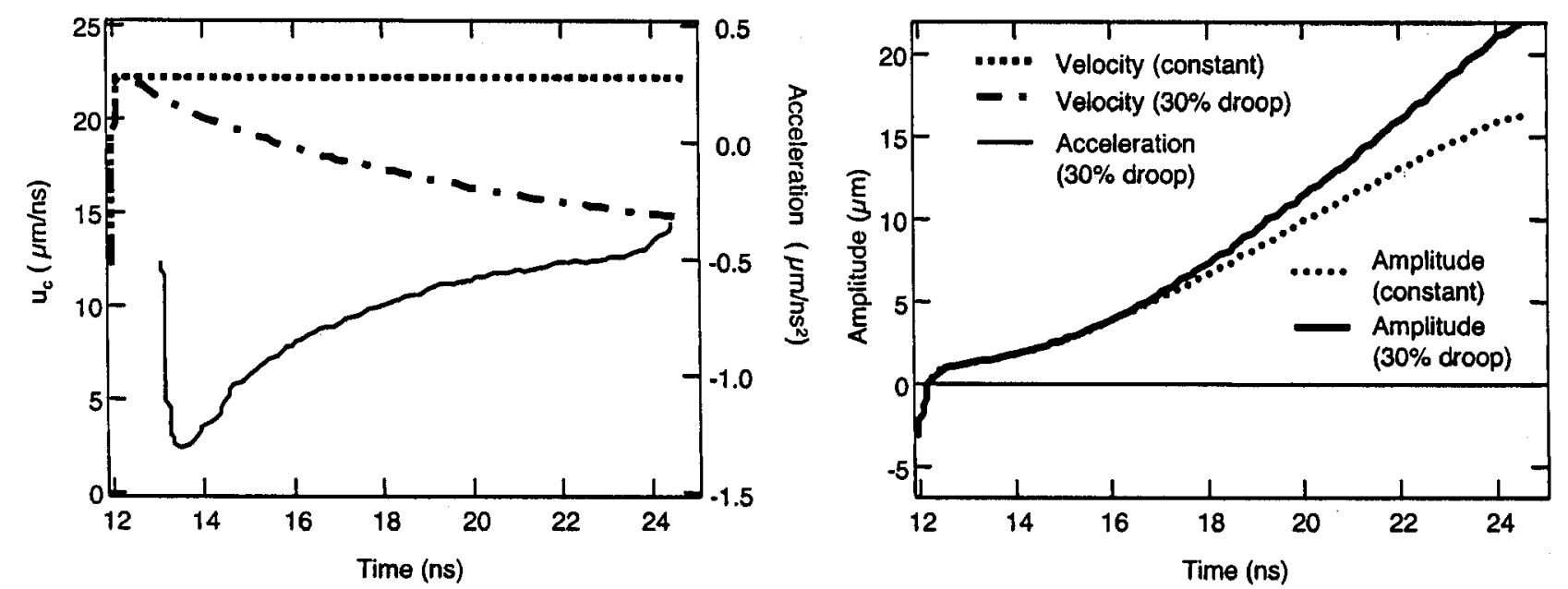
Figure 4.

a)

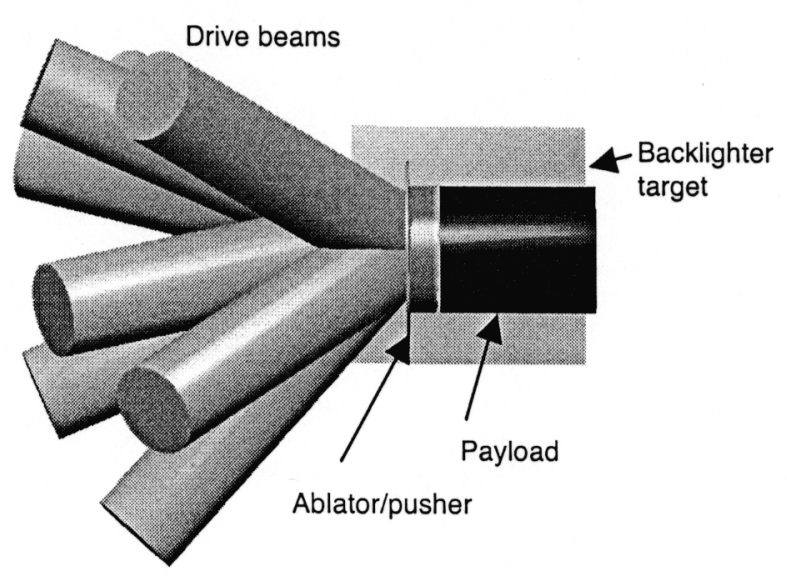

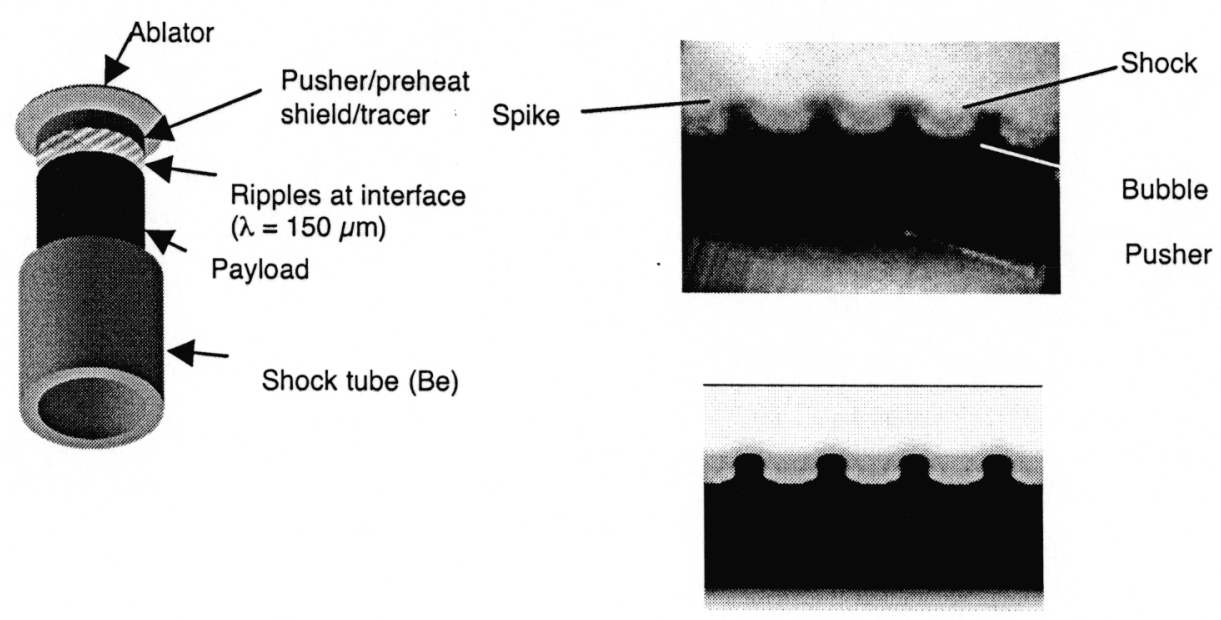


Figure 5

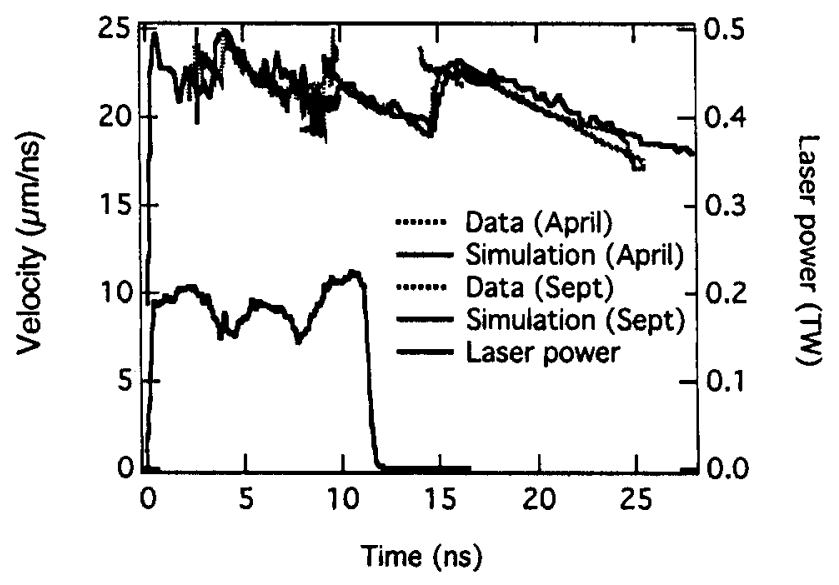


Figure 6.

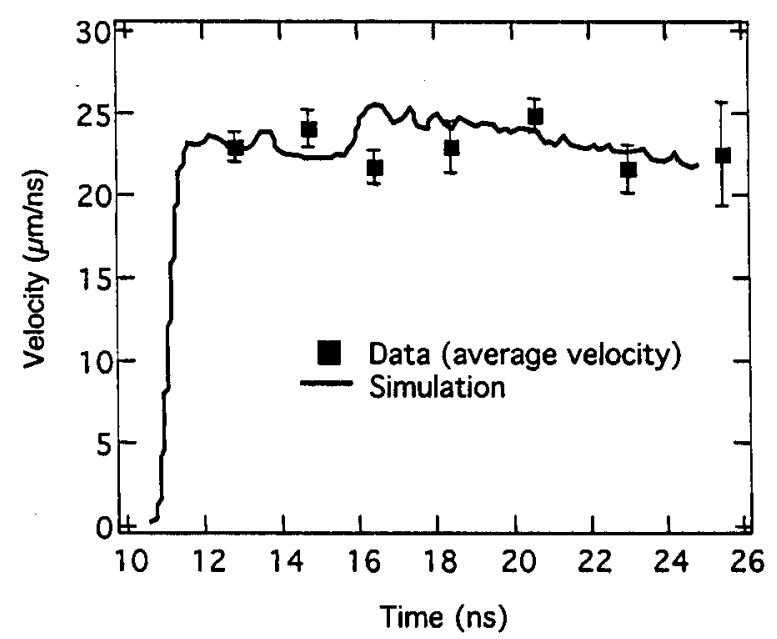


Figure 7.

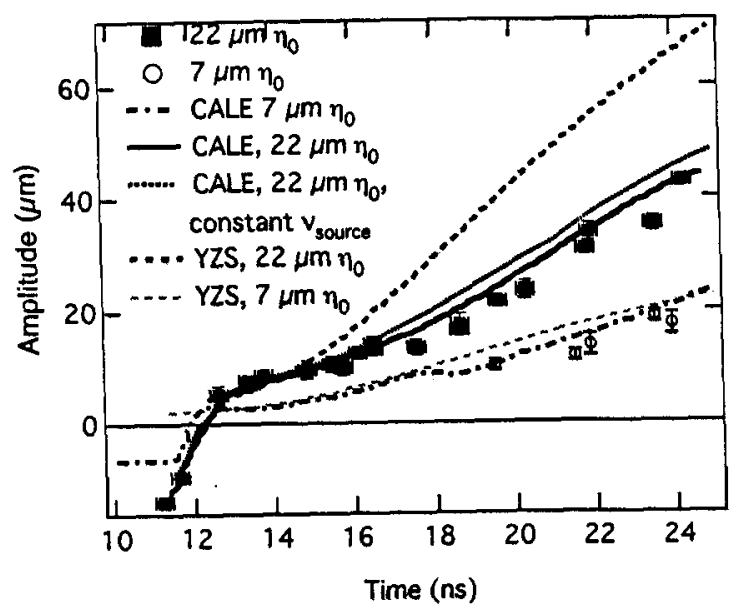

sgg-281002-01 
Figure 8

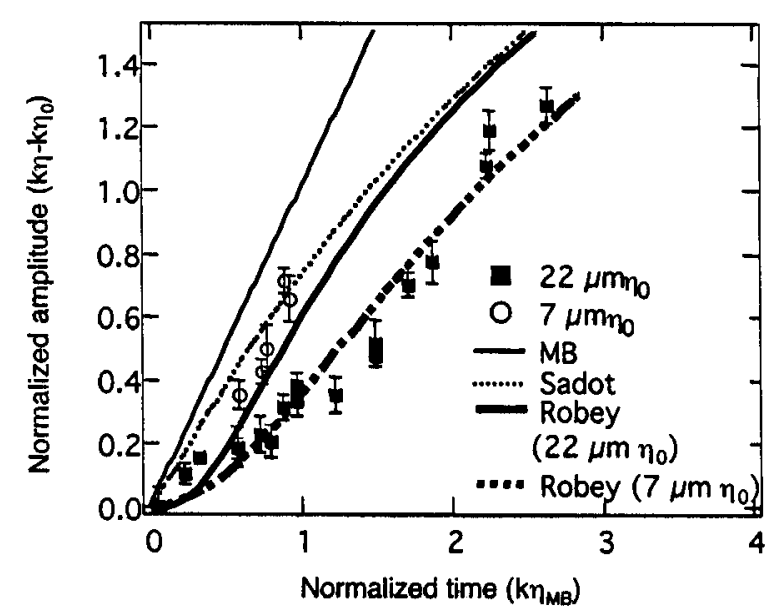

